ungsplans an sowie arrondierend abschließende Ausführungen zu den bauordnungsrechtlichen Anforderungen der Abstandsflächen und des Verunstaltungsgebots

Als formelles Recht der Anlagenzulassung schildert der Autor sodann im folgenden Kapitel das immissionsschutzrechtliche Genehmigungsverfahren für Windenergieanlagen (,an Land“), insbesondere das Genehmigungserfordernis und das Genehmigungsverfahren. Es schließt sich Exkurse zum Repowering von Windenergieanlagen und zu Offshore-Anlagen an, die vergleichsweise knapp ausfallen.

Schließlich widmet sich der Autor ausführlich den Rechtsschutzfragen. Unterteilt nach Bauherren, Nachbarn, Gemeinden, Nachbargemeinden, Dritten im Hinblick auf Offshore-Windenergieanlagen sowie ,,anerkannten Naturschutzvereinigungen“ schildert er die unterschiedlichen Rechtsmittel und Angriffspunkte, so auch die novellierte Verbandsklage nach dem Umweltrechtsbehelfsgesetz. Nach Auffassung von Gatz lassen sich nachwievor keine unmittelbar klagefähigen Rechte aus der FFH- oder Vogelschutz-Richtlinie herleiten.

Besonders für einen schnellen Überblick geeignet erscheinen die sich daran anschließenden wesentlichen Ergebnisse zu jedem Kapitel, die knapp und verständlich zusammenfasst werden.

Abschließend trifft der Autor Handlungsempfehlungen zur Standortsteuerung für Windenergieanlagen im Außenbereich durch Flächennutzungs- und Raumordnungsplanung. Gerade diese Handlungsempfehlungen sind sehr $\mathrm{zu}$ empfehlende Lektüre für die Planungsbehörden und alle sonst Beteiligten. Auf 25 Seiten werden alle aus Sicht des Autors wesentlichen Anforderungen zusammengefasst und bedeutsame Empfehlungen für die Praxis getroffen. Zwa wird man diese Empfehlungen nicht als ex cathedra gesprochene Leitsätze zu bewerten haben, aber angesichts ihres aktuellen Rechtsprechungsbezugs wird es mehr als hilfreich sein, die Handlungsempfehlungen in der fachlichen Praxis entsprechend zu beherzigen.

Das Werk von Gatz erscheint angesichts seiner präzisen Darstellungen und Aktualität als hervorragende Handreichung für den Praktiker und äußerst gelungene Zusammenfassung der rechtlichen Problemstellungen und ihrer jeweiligen Behandlung durch die Rechtsprechung. Die Lektüre ist wärmstens zu empfehlen. Der Vorteil gegenüber der detailreicheren Kommentarliteratur zu den einzelnen bei Gatz abgehandelten Rechtsgebieten liegt auf der Hand. Gatz liefert in einem kompakten Buch einen gehaltvollen Überblick zu allen relevanten Themenbereichen im Hinblick auf bauplanungs- und genehmigungsrechtliche Fragestellungen zur Onshore-Windenergie, der sich auf die wesentlichen aktuellen Rechtsfragen der Verwaltungs- und Gerichtspraxis konzentriert.

DOI: $10.1007 / s 10357-014-2695-3$

\section{Wolfgang Haber, Walter Bückmann: Nachhaltiges Landmanagement, differenzierte Landnutzung und Klimaschutz} gemeinschaft für Gesellschaft, Umwelt und Siedlung des Universitätsverlages der TU Berlin, Band 16, Berlin 2013 Universitätsverlag der TU Berlin, 407 S. ISBN 978-3-79832620-0 (print), ISBN 978-3-7983-2621-7 (online).

Die Autoren haben sich zum Ziel gesetzt, einen Weg zur politischplanerischen Realisierung nachhaltigen Landmanagements zu erarbeiten dessen Lösung sie mit der Anwendung eines Konzeptes der „Differenzierten Landnutzung" erreichen wollen. Eine differenzierte Landnutzung, die, wie sie diese beschreiben ,,außer der Biodiversität auch die unbelebte Natur mit Klima, Relief und Gewässern, sowie die strukturelle Vielfalt der Landschaft einbezieht und nicht zuletzt auch dem Bodenschutz dient".

Zunächst widmen sie sich die Autoren in den Kapiteln 2, 3, und 4 sehr ausführlich (und recherchieren dabei ausgesprochen gründlich und umfassend) den theoretischen Grundlagen der Nachhaltigkeit und der Sinnhaftigkeit des Begriffsinhaltes sowie der nachhaltigen Entwicklung im Besonderen. Theoretische Literatur und politisch/ praktische Programme werden dabei gleichermaßen einer systematischen Analyse unterzogen. Das Kapitel zum nachhaltigen Landmanagement ist sehr eindrucksvoll und verständlich geschrieben.

Professor Dr. Dr. Hans Günter Barth,

Kusadasi, Türkei
Die Abbildungen im Buch sind zumeist einprägsam, lockern den ansonsten eher trockenen und nüchtern gehaltenen Text auf und tragen viel zur besseren Lesbarkeit bei. Herausheben möchte ich dabei besonders das Schema zu den Begriffsinhalten des Landmanagements.

Mit ihrer gründlichen Studie der Umweltliteratur kommen sie so manchen Ungereimtheiten auf die Spur, was mit dem bemerkenswerten Satz zusammengefasst wird, dass es , unglaublich“ “ist ..., ,wie viel barer Unsinn ... in der offiziellen Umweltpolitik (allerdings nicht nur in dieser) verbreitet wird“"(S. 176)

In den Kapiteln 5, 6 und 7 wird dann nachhaltiges Landmanagement im Kontext mit Bodenschutz, Gewässerschutz und Klimaschutz diskutiert. Dem folgt dann schließlich in Kapitel 8 eine Auseinandersetzung mit der Relevanz politischer Programme für das Landmanagement (D und EU). Im Ergebnis gipfelt das in der ernüchternden Erkenntnis, dass alle in Betracht zu ziehenden Programme nicht brauchbar sein können, weil sie nach Kenntnis der Autoren „großenteils auf unzureichender Kenntnis der Situation“, „Beschönigen“ der Tatsachen, ,,mangelndem politischen Willen" zur Umsetzung u. a. basieren.

Die Lösungsvorschläge der Autoren kann man dann nach 320 Seiten Lektüre von Theorie und relevanten Gesellschafts- und Wissenschaftsbereichen, auf 19 Seiten kompakt dargestellt nachlesen. Dazu wird das von W. Haber schon in den 70er Jahren des 20. Jhdt. entwickelte Konzept „Differenzierte Boden- bzw. Landnutzung“ (DLN) aufgegriffen und näher erläutert. Der Grundgedanke der DLN läuft darauf hinaus, dass Nachteile großflächiger, gleichartiger Landnutzung vermindert werden durch Beachtung von sog. Belastungsregeln.

Ist das Buch bis jetzt eher gekennzeichnet durch theoretisch-abstrakte Erörterungen und Analysen, so erfährt man jetzt wohltuend konkret, wie denn DLN tatsächlich umgesetzt werden soll. Es werden dabei 2 Grundregeln aufgestellt, die beide mit konkreten Zahlen verbunden sind. Zum einen soll für die einzelnen Flächen einheitlicher Agrarnutzung eine Obergrenze von 25 ha eingehalten werden. Das zielt vor allem auf Minderung der Belastungen hin.

Zum anderen soll in solchen Gebieten eine Fläche von mindestens $10 \%$ dem ökologischen Ausgleich dienen. Dem zur Frage der Stabilität von Ökosystemen informierten Leser ist dies im Grundsatz zwar verständlich, alleine die Autoren bleiben ihm eine empirisch-ökologisch wissenschaftliche Begründung des „Zahlenwerkes“ schuldig.

Zusammenfassend: Das vorliegende Buch stellt eine umfassende, sehr sorgfältige Zusammenstellung, theoretischer und programmatischer Erörterungen zur Nachhaltigen Landnutzung dar und ist insofern neu in der relevanten Fachliteratur.

Die beiden Autoren, einschlägig ausgewiesene Umweltwissenschaftler, versuchen dezidiert nachzuweisen, dass Nachhaltiges Landmanagement der wirksame Weg zur Umsetzung des Nachhaltigkeitsprinzips und Klimaschutzes ist. Das wird mit Bezug auf agrarisch und forstwirtschaftlich genutzte Flächen genauso nachgewiesen wie auch hinsichtlich der Beanspruchung der Flächen für Siedlungsnutzung drastische Einschränkungen gefordert werden auf der Grundlage wirksamer Rechtsnormen und aktiver (Raum)Planung, die Umweltgerechtigkeit, Dauerhaftigkeit und Zukunftsfähigkeit beachtet.

Nachhaltiges Landmanagement wird von Wissenschaft und in politischen Programmen seit geraumer Zeit gefordert, hier wird zum ersten Mal der wissenschaftlich begründete „richtige Weg“ beschrieben. Das Buch ist (nicht nur) deshalb sehr lesenswert.

Ohne dass es die Autoren explizit genannt haben, so haben sie doch mit ihrer Abhandlung die Thematik der ausgehenden 70er Jahre in der Raumordnungspolitik wieder aufgegriffen, in der es um deren mangelnde Effizienz und dabei besonders um die sog. Vorranggebiete als neues Politikkonzept ging. Eng verbunden damit waren die sog. Ökologischen Ausgleichsräume, die vor allem von K. Buchwald und W. Haber seinerzeit gefordert worden sind. War es damals noch sehr fraglich, ob die Landwirtschaft in absehbarer Zeit auf räumliche Vorrangfunktionen im Vorranggebiet reduziert werden kann, so stellte es sich bei der Funktionszuweisung ,Natur und Landschaft mit ökologischer Funktion“, z. B. als ökologischer Ausgleichsraum, noch ungleich schwieriger dar. So wurde hier vor allem auf wissenschaftlich noch nicht hinreichend gesicherter Fragen der flächenbezogenen Ökosystemforschung hingewiesen. Dazu wurde in diesem Buch jetzt ein wichtiger Beitrag geleistet.

$\mathrm{Ob}$ das aber dem Konzept der Differenzierten Landnutzung angesichts der vorherrschenden Paradigmen in der Politik jetzt nicht auch so ergehen wird wie seinerzeit dem Konzept der Vorranggebiete in der Raumordnungspolitik bleibt zumindest fraglich. Es bleibt zu hoffen, dass es von Politik und planerischer Verwaltung nicht nur als lästige wissenschaftlich Kritik wahrgenommen wird, zwar sehr gescheit und profund formuliert, aber doch ohne praktische Konsequenzen beiseite geschoben wird. 